\title{
Metastasis, interrupted
}

By Michael J. Haas, Senior Writer

Two studies have clarified the roles of two different proteins in metastasis, suggesting potential new therapeutic strategies to treat cancer. One study proposes agonizing the membrane protein $\mathrm{CD} 151$ to prevent cancer cells from mobilizing at primary tumors; ${ }^{1}$ the other suggests turning off special AT-rich sequence binding protein 1 (SATB1), a regulatory master switch that promotes cancer cell invasiveness and metastasis. ${ }^{2}$

Company researchers contacted by SciBX agreed that both papers uncovered important molecular mechanisms that drive metastasis, but they were less certain that CD151 and SATB1 are druggable targets. Druggability concerns aside, the companies suggested it might be easier to demonstrate outcomes in the clinic by targeting SATB1 rather than CD151.

\section{Not letting go}

In their paper in Cancer Cell, researchers at The Scripps Research Institute and The Italian Foundation for Cancer Research Institute for Molecular Oncology report that agonizing the membrane protein CD151 promotes tumor cell immobility at the primary tumor site and prevents the cells from migrating. ${ }^{1}$ The team was led by James Quigley, a professor of cell biology at Scripps.

This work built on previous in vitro studies conducted at the State University of New York at Stony Brook by Quigley and colleagues, showing that tetraspanin CD151 plays a role in tumor migration and metastasis. ${ }^{3}$

Tetraspanins are membrane proteins thought to anchor other proteins to the cell membrane. CD151, also known as tetraspanin 24, is a cell-surface signal transducer involved in cell development, activation, growth and motility. It complexes with integrins and other tetraspanins and is believed to regulate the functions of these proteins. CD151 is overexpressed in many cancers.

In the current paper, chick embryos and mice with fluorophorelabeled human tumors were injected with 1A5, an anti-CD151 mAb previously developed by Quigley at SUNY Stony Brook. ${ }^{4}$

Tumor cells were tracked with Innovascreen Inc.'s intravital imaging technology - a general term for methods that image live tissue, according to the company's CEO, John Lewis. He said Innovascreen has improved intravital technology by developing the chick embryo model-which extends the real-time imaging timeframe from hours to days-and by incorporating new fluorescence techniques and instrumentation to increase resolution and overall utility.
Quigley's team found that 1A5 prevented tumor cells from detaching and migrating into the bloodstream-a process called intravasation. 1A5 did not prevent tumor cells from leaving the bloodstream and entering new tissues (extravasation), but it did prevent them from migrating through those tissues afterwards.

The mAb had no effect on the motility of CD151-deficient tumor cells. Nor did the antibody have an effect on tumor growth and proliferation, although it prevented primary tumor cells from invading the surrounding tissue.

The findings indicate that $1 \mathrm{~A} 5$ stimulates the natural adhesionpromoting function of CD151, thereby inhibiting tumor cell motility and blocking the first step of metastasis.

Andries Zijlstra, spokesperson for the team, said the natural ligand of CD151 and the exact mechanism by which CD151 promotes adhesion are unknown. What is known is that CD151 interacts with integrin $\alpha_{3} \beta_{1}$ (VLA-3) and integrin $\alpha_{6} \beta_{1}$. Thus, a possible explanation is that CD151 regulates adhesion and migration by direct control of integrin function.

Formerly at Scripps, Zijlstra is now an assistant professor of pathology and cancer biology at Vanderbilt University.

The question is whether the CD151 findings can be translated into antimetastatic therapies, given the problems with designing clinical trials that show prevention of metastasis.

"It has long been hypothesized in the metastasis community that there should be some way to prevent tumor cells from leaving the primary tumor," said David Young, president and CEO of Arius Research Inc. "So it's interesting to find a molecule that regulates this process. But the relevance to drug development is quite challenging" because of the difficulties of testing whether a compound prevents metastasis.

"You are trying to prove a negative- that metastasis hasn't occurred," he said. "The population size and long follow-up time required to study an antimetastatic drug compound can be problematic."

Arius has two compounds in preclinical development: AR001, a mAb targeting CD44 on cancer stem cells, and AR002, a mAb targeting the signal transducer tumor-associated calcium signal transducer 2 (TACSTD2; TROP2). The company plans to start Phase I trials of both compounds to treat solid tumors in late 2008.

Another issue, said Young, is the lack of data on the mechanism by which CD151 promotes adhesion. "It might not be the culprit molecule. It might interact with something else that is the dominant player. CD151 might be the organizer factor, not the primary effector" of the adhesion process, he said.

"Also, they haven't shown the other half of it-increased survival in connection with decreased metastasis in the animals," Young said. Such a correlation could provide a measurable endpoint for clinical development and possibly show whether CD151 is indeed the governing molecule in the process, he said.

Andrew Mazar, CSO and SVP of R\&D at Attenuon LLC, thinks antiCD151 therapies may be too little, too late. "The problem here is that the research team is looking at a process that leads to intravasation," he said. "But by the time a primary tumor is macroscopically detectable, it may well already have intravasized and metastasized." 
Attenuon's ATN-161 is a five-residue peptide derived from fibronectin that targets integrin $\alpha_{5} \beta_{1}$ and integrin $\alpha_{v} \beta_{3}$. In mid-2007 the company halted a Phase II trial of the compound in intracranial malignant glioma because of the inconvenience of the i.v. formulation. Attenuon is reformulating the compound for subcutaneous injection and expects to re-enter the clinic within 18 months.

Tim Hoey, VP of cancer biology at OncoMed Pharmaceuticals Inc., thinks the findings would be difficult to develop clinically because targeting CD151 did not affect growth of the primary tumor. "If the antimetastatic agent didn't affect the primary tumor, it would be harder to follow its effects. You would have to do a trial around long-term survival or absence of metastasis-endpoints that take years to play out. That's quite a gamble of time and expense for proof of concept."

OncoMed's OMP-21M18 mAb against an undisclosed target on cancer stem cells is expected to start a Phase I trial in solid tumors in late 2008. The compound is partnered with GlaxoSmithKline plc.

"It is usually necessary to show some effect on the tumor," Young noted. "And an antimetastatic drug is not likely to be chosen as a first-line treatment for early-stage patients-even though they would most likely benefit from it."

Zijlstra acknowledged it is difficult to predict when metastasis will occur, but disagreed that targeting the CD151-mediated mobilization of tumor cells would have no effect on the primary tumor.

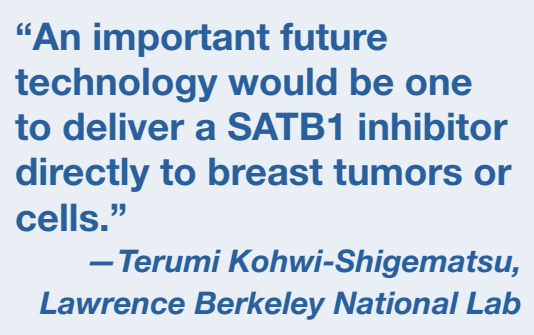

However, the consensus is that targeting SATB1 directly will still be difficult and could have far-reaching side effects.

SATB1 is a nuclear protein that regulates chromatin-the intertwined DNA and proteins that make up chromosomes. SATB1 forms cage-like structures that fold and remodel chromatin to coordinate gene expression and regulation. It is also expressed on normal, activated T cells.

Kohwi-Shigematsu and her team examined 24 breast epithelial cell lines and detected SATB1 expression only in cells known to be aggressive and metastatic. The team also detected SATB1 in aggressive types of human primary breast tumors, but not in adjacent, nonmalignant tissue.

"Normal epithelial breast cells do not express SATB1 at all," she told SciBX. "Many cancer cells do not express SATB1 either. SATB1 expression is closely associated with aggressive and metastatic cancer cells."

The team next showed that short hairpin RNA knockdown of SATB1 reversed the metastatic activity of metastatic breast cancer cells in vitro and in mice. They also showed that abnormal expression of SATB1 in mice with ordinarily nonmetastatic tumor cells induced the development of invasive, metastatic tumors.

Thus, the team concluded that expression of SATB1 was key to the metastatic phenotype of tumor cells. "Our results suggest that SATB1 is induced during cancer progression, and once SATB1 is expressed, cells metastasize," KohwiShigematsu said.

Gene expression profiling of breast tumor metastasis," he said. "It is also responsible for the local invasion of host tissue that characterizes a malignant tumor."

Thus, immobilizing tumor cells might prevent metastasis and revert the malignant phenotype of a primary tumor. "This is particularly important for invasive tumors of critical organs such as pancreatic and brain cancers," which are not metastatic in the classic sense because the tumor cells haven't migrated to distant sites, said Zijlstra.

Such tumors might benefit from a therapy like the anti-CD151 mAb, he said, because "these invasive structures are difficult, if not impossible, to remove surgically."

Zijlstra said the research team is investigating potential adhesion and de-adhesion partners for CD151. "We have found about 50 of them and are looking at which ones result in the immobile phenotype" in cancer cells and might provide better targets than CD151, he said.

The research team also is conducting a "study that correlates CD151 and its partners to disease progression and survival," Zijlstra said.

\section{Metastatic master switch?}

Meanwhile a paper in Nature by researchers at the University of California's Lawrence Berkeley National Laboratory and Fox Chase Cancer Center reports that SATB1 is expressed in breast cancer cells and deregulates the expression of many proteins, including cell adhesion molecules that enable tumor cell migration. ${ }^{2}$ The research team, led by Terumi Kohwi-Shigematsu, senior scientist at Lawrence Berkeley, suggests that SATB1 inhibitors could be used to treat or even prevent metastatic breast cancer.

Companies and institutions contacted by SciBX said the results on SATB1 may hold greater promise than CD151 for drug development. cells revealed that SATB1 upregulated or downregulated more than 1,000 genes. The largest proportion corresponded to genes that code for cell adhesion molecules.

Among the upregulated molecules were three that are often seen in invasive breast cancer: OB-cadherin (cadherin-11), VE-cadherin (CD144; cadherin-5) and N-cadherin. Among the downregulated adhesion molecules was the junction protein and tumor suppressor E-cadherin, which helps normal cells stay in place.

Taken together, Kohwi-Shigematsu said the data strongly suggest that breast cancer metastasis results from expression of SATB1, which in turn alters the expression profile of many genes to promote metastatic activity and tumor growth. Thus, targeting SATB1 in tumor cells could reverse the aggressive phenotypes of metastatic breast cancer, she said.

Arius's Young was interested in the correlation Kohwi-Shigematsu's team found between SATB1 and tumor growth and metastasis, but would need to know more about the role SATB1 plays in normal tissues before deciding whether it is a druggable target. The researchers "haven't done a lot to characterize the expression of SATB1 in normal cells and tissues," which raises potential toxicity issues when targeting the protein, he said.

Young added that the genetic regulatory functions of SATB1 made it a difficult target. "A lot of downstream target genes are regulated by SATB1. That makes for a lot of potential-and unwanted-consequences in targeting such a 'master switch"' he said. "A mass of targets would be affected. This might be too general of an effect."

Young suggested one or more of the downstream proteins governed by SATB1 might make better targets against metastasis. But Kohwi- 
Shigematsu noted that downstream proteins have functions in normal cells and targeting them also could result in toxic side effects.

"An important future technology would be one to deliver a SATB1 inhibitor directly to breast tumors or cells," efficiently destroying only metastatic cells, she said.

OncoMed's Hoey noted that a nuclear transcription factor like SATB1 would also be technically difficult to target.

"Non-enzymatic intracellular proteins have been difficult to target with small molecules," he said. "Apart from compounds targeting nuclear receptors such as peroxisome proliferation-activated receptors, the technology seems a long way from producing a drug without some kind of breakthrough."

Hoey agreed that downstream molecules regulated by SATB1 might be more accessible.

Attenuon's Mazar concurred that SATB1 was a more attractive target than CD151 for metastasis, in part because targeting SATB1 did not depend on catching metastasis before it occurred.

However, like Hoey, Mazar was not sure how to target the nuclear protein. "Is there a regulatory site on SATB1 that can be targeted with a small molecule to prevent its transcriptional activity?" he said.

"The paper goes a long way toward target validation, but I'm not sure whether SATB1 is necessarily a druggable target-many proteins aren't," Mazar said.

\section{Pulling it all together}

Zijlstra was interested in how his team's and Kohwi-Shigematsu's papers intersected at the point of cell adhesion processes, noting that SATB1 downregulated E-cadherin, a protein with a "rigid adhesion profile" that prevents normal cells from moving, he said.

"Cancer cells overcome these rigid adhesions by turning on EpCAM [epithelial cell adhesion molecule], CD44 and other more 'promiscuous' adhesion molecules," some of which the Nature paper shows are upregulated by SATB1, Zijlstra noted. That, he said, allows the cancer cells to make and break adhesive interactions more readily, giving them greater motility than normal cells.

Zijlstra said his team's work suggests that exogenous promotion of tumor cell adhesion-by targeting CD151 or its adhesion partners-might counter the increased motility that SATB1 induces endogenously.
Beyond that, both papers present an exciting perspective on metastasis, he said. "The papers show that tumor cells become malignant in an epigenetic manner," he said. "Because tumor cells are not genetically mutated, but only have changes to expression and activation, they retain their susceptibility to reversion of malignancy. If we can disrupt these processes with the right drugs, we can actively reverse malignancy. In other words, we can still normalize the cancer cells."

Going forward, Kohwi-Shigematsu and her team have several experiments planned.

"First we wish to determine what turns SATB1 on in breast cancer," she said, although her team has not identified any factors so far. "We are also looking for SATB1 expression in other cancer types, to see if SATB1 could be a more generic marker for metastasis."

She said the team is already considering whether to carry out experiments correlating decreased metastasis with increased survival, as Young would like to see, but declined to disclose any details.

\section{REFERENCES}

1. Zijlstra, A. et al. Cancer Cell; published online March 12, 2008; doi:10.1016/j.ccr.2008.01.031

Contact: James P. Quigley, Department of Cell Biology, The Scripps Research Institute, La Jolla, Calif. e-mail: jquigley@scripps.edu

2. Han, H.-J. et al. Nature; published online March 12, 2008; doi:10.1038/nature06781

Contact: Terumi Kohwi-Shigematsu, Life Sciences Division, Lawrence Berkeley National Laboratory, University of California, Berkeley, Calif. e-mail: terumiks@|bl.gov

3. Testa, J. et al. Cancer Res. 59, 3812-3820 (1999)

4. Brooks, P. et al. J. Cell Biol. 122, 1351-1359 (1993)

COMPANIES AND INSTITUTIONS MENTIONED

Arius Research Inc. (TSX:ARI), Toronto, Ontario, Canada

Attenuon LLC, San Diego, Calif.

Fox Chase Cancer Center, Philadelphia, Pa. GlaxoSmithKline plc (LSE:GSK; NYSE:GSK), London, U.K. Innovascreen Inc., New Glasgow, Nova Scotia

The Italian Foundation for Cancer Research Institute for Molecular Oncology, Milan, Italy

Lawrence Berkeley National Laboratory, University of California, Berkeley, Calif.

OncoMed Pharmaceuticals Inc., Redwood City, Calif.

The Scripps Research Institute, La Jolla, Calif.

State University of New York at Stony Brook, Stony Brook, N.Y.

Vanderbilt University, Nashville, Tenn. 